\title{
Ultrasound Assisted Spinal Anesthesia: A Safety Procedure or a Lost of Time?
}

\author{
Sessa Flaminio ${ }^{1}$, Levantesi Laura ${ }^{1}$, De Santis Renata ${ }^{2}$, Nicosia Luca ${ }^{3}$ and De Cosmo Germano ${ }^{1 *}$ \\ ${ }^{1}$ Department of Anestesia, Rianimazione e Terapia Intensiva, Fondazione Policlinico University Agostino Gemelli IRCCS, Roma (RM) \\ Italy \\ ${ }^{2}$ Department of Anestesia, Ospedale San Pietro Fatebenefratelli, Rome (RM) Italy \\ ${ }^{3}$ Advanced Radiation Oncology Departement, IRCCS Sacro Cuore Don Calabria Hospital, Negrar (VR) Italy
}

*Corresponding author: De Cosmo Germano, Fondazione Policlinico Universitario A Gemelli IRCCS, Università Cattolica del Sacro Cuore, Roma

\section{ARTICLE INFO}

Received: 幽 November 18, 2019

Published: 幽 November 27, 2019

Citation: Sessa Flaminio, Levantesi Laura, De Santis Renata, Nicosia Luca, De Cosmo Germano. Ultrasound Assisted Spinal Anesthesia: A Safety Procedure or a Lost of Time?. Biomed J Sci \& Tech Res 23(2)2019. BJSTR. MS.ID.003880.

\section{ABSTRACT}

The role of ultrasound (US) in central neuraxial blocks is often underestimated for the difficulty in ultrasound visualization. The utility of ultrasound technique is more evident in expected difficult cases for the relative efficacy of blind techniques. Studies with larger sample size are necessary to improve spinal ultrasound use in neurassial anesthesia.

\section{Introduction}

The role of ultrasound (US) in central neuraxial blocks has often been underestimated, partly due to the relative efficacy of blind techniques (based on the exploration of the classic anatomical landmarks), partly due to the difficulty in the ultrasound visualization due the acoustic window produced by spine bony structures. Nevertheless, in the last decade, ultrasound usage in neurassial anesthesia has aroused greater interest, due to its great usefulness [1]. The first description of an echo-assisted lumbar puncture dates to 1971. More recently, neurassial ultrasound was used both as a pre-procedural aid and for real-time needle placement (eco-guided technique). Imaging in the adults is possible only through the interlaminar space between adjacent elements. Combining various ultrasound scans allows to study the patient's vertebral column, exploring the anatomical structures to be crossed and recording relevant parameters for the anesthesiologist [2-4] such as intervertebral levels, depth of the epidural and subarachnoid space and to trace the optimal spinal needle entry point. The utility of ultrasound technique is more evident in expected difficult cases, when only superficial anatomical landmarks are poor, such as obesity or excessive thinness, and previous spinal surgery, or in case of deformity of the vertebral column [5]

Conventional palpation of the surface anatomy may be insufficient for an exact neurassial anesthesia [6]. For example, in elderly, midline canal access may be more difficult due to narrowing or calcification of the interspinous space, heterotopic ossification of the interspinous ligaments, or joint hypertrophy. Most of the evidence related to neurassial ultrasonography are derived from obstetric anesthesia performed in limited specialized centers [7]. Those evidences show that ultrasound may reduce attempts number, can accurately predict epidural space depth and can therefore significantly improve the success rate of anesthesia, even in young specialists. Similarly, to other loco-regional anesthesia techniques, the real-time ultrasound-guided spinal needle penetration in the subarachnoid space requires technical ability 
and has a learning-curve, in order to maneuver coherently the ultrasound probe and the needle. The ultrasound-guided technique consists of an accurate pre-procedural patient's back scan and has usually a shorter learning-curve. Ultrasound-guided single shot subarachnoid anesthesia is less studied. An observational study in orthopedic patients showed that ultrasounds may accurately predict dura mater depth [8].

Moreover, pre-procedural US has been decisive in clinically difficult situations, such as obesity, kyphoscoliosis and cases of previous spinal surgery $[9,10]$. The echo-guided subarachnoid anesthesia has been described in technically difficult and prone position patients. via the Taylor approach [11]. Perlas et al. [12,13], answered some fundamental questions about the US approach to neurassial blocks. First point was the US-related block effectiveness and quality. Vallejo et al. randomized 370 parturient undergoing epidural analgesia to receive US-guided versus blind technique; the study was performed by both expert anesthesiologists and young in training [14]. The failure rate was significantly lower in the USguided group. The second aspect was the forecast of difficulties. The difficulty of a neurassial block is quantifiable through two main parameters: the number of attempts to introduce/realign the needle and the time to block. Of the two, we consider the number of attempts/realignments the most important factor, since a complicated insertion of the spinal needle is an independent risk factor for possible complications as vascular puncture, paresthesia, hematomas, long term neurological damage $[15,16]$.

In a randomized trials US usage reduced the number of spinal needle movements by $50 \%$ and significantly increased the first attempt success rate [17]. As a further support to the safety with US-technique, Shaikh et al. reported a reduced rate of realignment and spinal punctures for neurassial procedures, including epidural catheterizations, in a 2013 meta-analysis [18]. Several case reports have also described neurassial blocks successfully carried out in markedly obese patients, elderly, patients with spinal deformities and with a history of vertebral surgery (once considered a contraindication to the subarachnoid procedure), by means of US support [19-21]. A randomized controlled trial conducted on 120 patients with difficult response points demonstrated, as a primary endpoint, a greater success rate at the first puncture attempt and needle redirects with US-guided technique. Therefore, vertebral column US by an expert operator ensures a greater ease of block execution, particularly in patients where a certain degree of technical difficulty is already foreseen.

After adequate training, US technique may provide valuable predictive difficulty information before procedure begins in less experienced operators. Two small studies examined the learning curve during lumbar tract US training. Margarido et al. [22] recruited 18 anesthesiologists with no previous experience in spinal US, providing them with a complete training period, which included reading material, educational videos, a 45-minute lesson and a 30-minute practical workshop. After 2 weeks, Authors evaluated subject's ability to identify lumbar intervertebral spaces, to trace a landmark for needle insertion and to measure the depth of the intrathecal space. Only five (27\%) subjects had acquired a competence regarding the identification of intervertebral spaces; no one has demonstrated competence in the other two tasks. Halpern et al. [23], evaluated the learning curve of two anesthesiologists with no previous experience in ultrasonographic imaging of the lumbar spine, after training on five patients each.

The competence (precision> $90 \%$ ) was reached by a colleague, after having performed the procedure in 22 patients; the other colleague, after 36 patients. Another point to consider is the prediction of the path through the anatomical planes. Knowing in advance the epidural or intrathecal space depth in $\mathrm{cm}$ from the skin allows not only to choose a needle of appropriate length, but also to obtain information about patient anatomical variants. The correlation between US depth and that detected with needle insertion was evaluated in several studies [2426]. Based on measurements conducted in different ultrasound views, the correlation was excellent in all studies. Albeit rarely, an incorrect intervertebral spaces identification can lead to serious complications, such as spinal cord injury [27]. In adults (unlike in pediatric patient) the medullary cone is hardly appreciated, due to its depth and to a more restricted acoustic window at the vertebral canal level. In this case, US can identify intervertebral levels more accurately, starting from the sacral junction (clearly distinguishable from other spaces) and identifying the spinous processes and/or laminae in a cranial direction.

This scanning mode is more accurate than the one with the use of intercaecal line as external anatomical reference point. Schlotterbeck et al. [28,29], have found that, in case of discordance between real and US anatomical landmarks, the intervertebral space was generally lower than the one identified by US. In contrast Locks et al. [30] observed that, the real level was cranial than the US reference. US is not unerring. When compared with other imaging modalities, such as MRI or CT, US can correctly identify structures in only $68-76 \%$ cases. Nevertheless, the US inaccuracy degree is considerably lower than the manual palpation of anatomical landmarks in the order of one intervertebral space versus 3 intervertebral spaces, respectively [31]. Moreover, it should be considered that US errors are operator-dependent and are therefore more likely at the beginning. Precision level greater than $90 \%$ can be achieved with an appropriate training and experience; mannequins and phantoms have proved to be useful for the training of young anesthesiologists or specialists with little experience in spinal US [32,33].

The most common errors usually derive from the misidentification of the L5-S1 junction or lumbosacral junction anomalies, which occur in approximately $12 \%$ of the general population. The most common anatomical variant is the 
sacralization of L5 in which L5 is fused to the sacrum and one or both transverse processes are involved. Less frequently, S1 can resemble a lumbar vertebra (lombarization). In this sense, counting vertebrae from D12, identifying the twelfth rib through US, to the lumbo-sacral junction, represents a good strategy. Kim et al. [34] have shown that the distance between the medullary cone and the Tuftier line is similar both in patients with lumbosacral junction abnormalities and in those without. In conclusion, the vertebral counting starting from the lumbosacral junction seems an adequate and sufficiently safe US technique. The most common pitfall of the US approach is the poor image quality with difficulties in identifying deeper structures such as the epidural space, dura mater, intrathecal space and anterior complex in markedly obese patients and in the elderly.

Furthermore, in obese patients there may be an image attenuation due to the abundance of soft tissues Moreover, it can be an image aberration due to the different sound propagation speed through irregular and deformed adipose tissue [35]. However, the identification of spinous processes and the midline is almost always possible, even in these cases. In the elderly, ligaments ossification and facet joints hypertrophy cause a narrowing of the interspinous and interlaminar spaces. In skinny patients, prominent and sharp spinous processes could reduce correct probe-skin contact and determine a poor image quality. In such patients, the oblique view in paramedian sagittal orientation is the best choice. For what concerns the choice of the US probe, a convex at low frequency (2-5 MHz) is suggested. It can obtain a wider field and a deeper US penetration and can improve the recognition of anatomical structures and image quality [36]. An initial depth of 7-8 cm is appropriate for most patients, bearing in mind that depth, focus and gain must be adjusted by the operator during the scanning process to produce a high-quality image (Figure 1). Spine can be studied with five basic US views [37]:

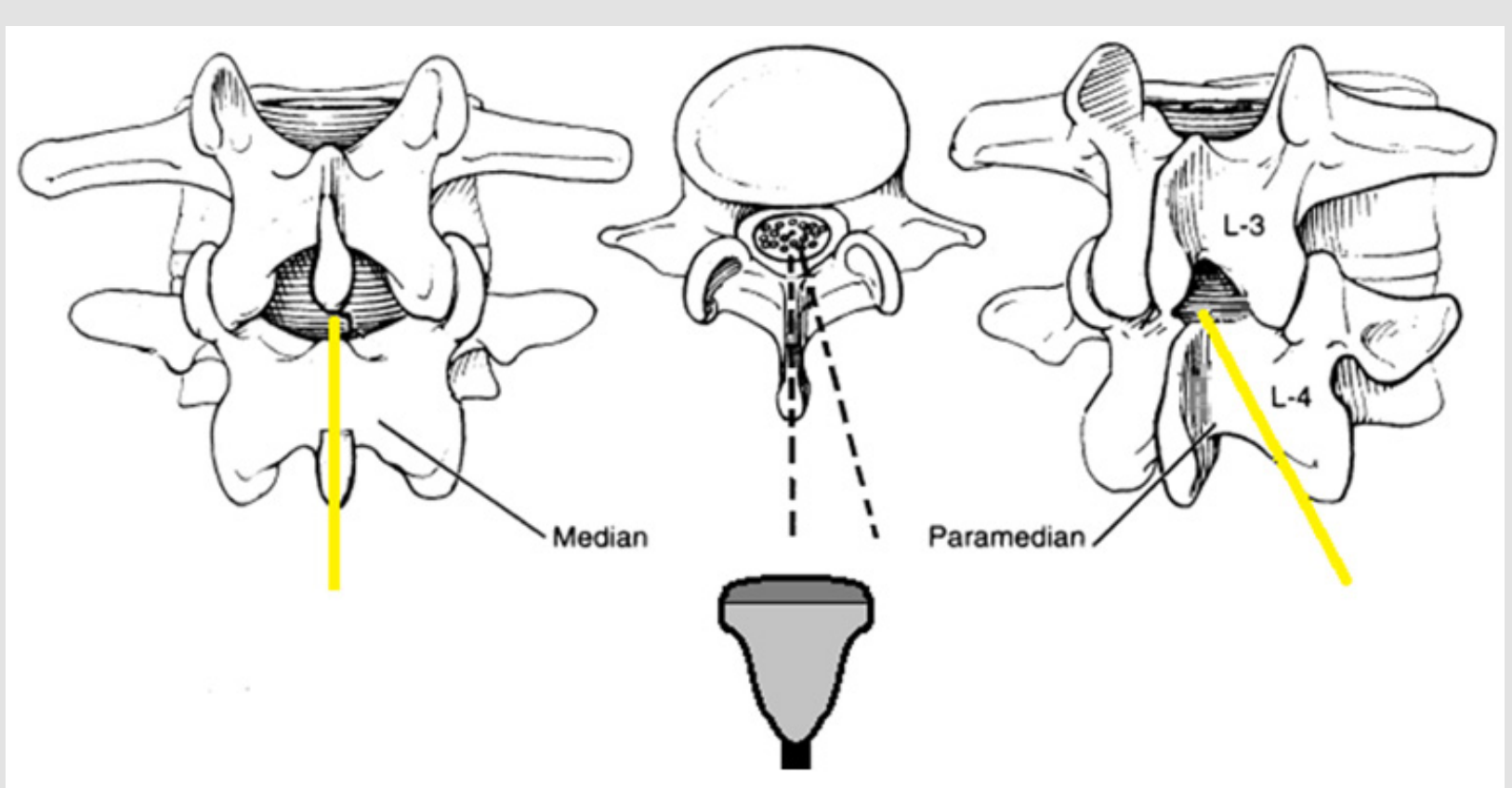

Figure 1.

\section{Visualization of Transverse Processes in Sagittal Paramedian}

The probe is positioned $3-4 \mathrm{~cm}$ lateral to the midline, just above the sacrum. In this image, the transverse processes of the subsequent lumbar vertebrae are visible as small hyperechoic curvilinear structures, the "trident sign". The psoas muscle is visible between the acoustic shadows, deeply to the transverse processes.

\section{Visualization of Articular Processes in Sagittal Paramedian}

The probe is slides medially, until it meets a hyperechoic line of "humps" corresponding to the facet joints (at a lower depth than the transverse processes) of two adjacent vertebrae.

\section{Visualization in Oblique Sagittal Paramedian}

Once the transverse processes have been identified, the probe is tilted to put the beam in a lateral-medial direction, towards the midline. The hyperechoic sloping lumbar vertebrae laminae form a "saw-tooth" pattern among which the paramedian interlaminar spaces can be identified. Through the spaces, the following structures can be appreciated (from the most superficial to the deepest): yellow ligament, epidural space, posterior dura mater, intrathecal space, dura mater anterior, posterior longitudinal ligament and posterior vertebral body. The yellow ligament, the epidural space and the posterior dura often appear as a single linear hyperechoic structure, which is globally defined posterior 
complex. If the patient's anatomy allows it, with small sliding and tilting probe movements, the yellow ligament and posterior dura can be recognized as two hyperechoic lines separated from the posterior epidural space, hypoechoic due to adipose tissue.

The intrathecal space is uniformly hypoechoic, although the cauda equina and the filum terminal may appear as hyperechoic strips. The anterior dura mater, the posterior longitudinal ligament and the posterior part of the vertebral body or the intervertebral disc appear as a single linear hyperechoic structure, called anterior complex, often indistinguishable in adult patients. The interlaminar space size can be estimated from the length of the anterior complex or from the posterior complex, thus predicting block technical difficulty. The posterior complex-skin distance can indicate the target depth for the spinal needle. In order to identify intervertebral spaces, while in sagittal oblique paramedian probe position slide in caudal direction, until visualizing the horizontal hyperechoic line of the sacrum. In this way, the space between the sacrum line and the "tooth" of the L5 lamina corresponds to the L5-S1 intervertebral space (L5 lamina is typically thinner than the other lumbar laminae). The other intervertebral spaces are practically identified, cranially enumerating them starting from the lumbo-sacral junction and marking with a demographic pen.

\section{Visualization of the Spinous Processes in Transversal}

Once completed the paramedian sagittal plane examination, the probe is rotated $90^{\circ}$ in a transverse orientation and then moved to the midline. If the probe is above a spinous process, this will appear as a superficial hyperechoic line above a hypoechoic shadow. The lamina is visible on both sides of the spinous process as a hyperechoic wing, while all the other structures of interest are covered by shadow cones generated by the bony tissue.

\section{Visualization of the Interlaminar Space in Transversal}

Slide the probe in the cranial or caudal direction starting from the spinous process, align the US beam with the interspinous and interlaminar spaces, to visualize the vertebral canal in transverse orientation. Typically, the acoustic shadow generated by the spinous process gives way to an iperechoic vertical line (the interspinous ligament surrounded by the erector muscles) and, more deeply, two parallel hyperechoic lines separated by a hypoechoic space (posterior and anterior complex separated by intrathecal space). Depending on the width of the interspinous space and the projection angle of the spinous processes, the probe can be tilted to improve the image quality of the vertebral canal. In this case, unlike the oblique sagittal paramedian orientation the yellow ligament and the posterior dura are rarely visible as distinct structures and may not be occasionally visible. The poor visibility of the posterior complex may depend on a reduced acoustic window, or on small gaps inside the yellow ligament.

When the beam passes through the vertebral canal, the vertebral body complex is visible and the interlaminar space has been correctly identified [38]. The transverse processes and the articular processes are additional reference points, since they lie approximately on the same level as the interlaminar space, resulting useful for the needle orientation in difficult anatomical cases. The midline and the interlaminar space can therefore be marked on the patient skin at the midpoint of each side of the convex probe (long and short, respectively). The intersection of the lines passing for these two landmarks indicates therefore the optimal puncture site for a median approach, both for subarachnoid anesthesia and for other neurassial blocks. Furthermore, the spinal needle angle can be estimated from the probe tilting degree necessary to obtain a good interlaminar view. Our experience concerns orthopedic surgery. Locoregional anesthesia is the gold standard, due to the many advantages in terms of intra and postoperative pain control, and to the mortality and morbidity reduction.

The US-guided technique requires a first US look on a sterile field, before proceeding to the puncture and subarachnoid anesthesia. Progress in the locoregional field has improved efficacy and safety of peripheral and central nerve blocks. In expert hands, loco-regional techniques can result in greater patient comfort, reduced hospitalization time and general anesthesia-related complications, with early patient mobilization in the postoperative [39]. Subarachnoid anesthesia is widely used in major orthopedic surgery, such as hip and knee replacement. Although representing a routine anesthesiologic technique, is not without risk and complications $[40,41]$. Spinal US might be more widely used and not limited to case of difficult anatomical spine exploration. After positioning the patient in a conventional way (sitting or in lateral decubitus), we use the convex probe (low frequency, from 2 to $5 \mathrm{MHz}$ ) coated with a sterile probe cover, in a central position. The first step is midline recognition. We start the examination at the height of interspinous line, in transverse orientation. In this position by aligning the probe, we obtain a symmetrical image, in which the spinous process or the interlaminar space are located at the center of the.

By sliding the probe in the cranial direction, we trace a landmark on the skin with a demographic pen corresponding to the center of the image itself. Subsequently, it will be useful to identify the lumbo-sacral junction. The probe is $90^{\circ}$ rotated to obtain a paramedian sagittal view of the vertebral laminae. After identifying a lamina at the level of the iliac crests, the probe is slided until it meets the hyperechoic line of the sacrum. An anterior complex should be identified between the sacrum and lamina of the fifth lumbar vertebra. Once the L5 lamina is identified, the probe is rotated transversely and moved cranially to L3-L4, counting the intervertebral spaces starting from the lumbo-sacral junction. A slight tilt during the slide is necessary to improve the appearance of anterior e posterior complexes encountered along the way. Having reached the desired level, the structures of interest are identified: the first is the dura mater in the posterior complex, this step is necessary to measure the centimeters from the skin. The depth of the dura mater might be overestimated whether the anterior complex is confused with the posterior complex. 
The degree of tilting will indicate the needle angle at the time of puncture. At this level, while in transversal orientation, four skin landmarks are marked on the midpoints of each probe side. The intersection of the four points in a cross shape is the needle insertion point. The vertical line should correspond to the midline originally indicated. To be mentioned, after US scan patient must not move, otherwise anatomical skin landmarks might significantly change. To ensure the positioning, the probe is reapplied to the center of the cross to have a good view of the anterior complex. The examination can be integrated with the oblique sagittal paramedian visualization, tilting the probe medially, with the aim of highlighting the posterior and anterior complexes through the interlaminar space. This visualization is of greater help in the USguided subarachnoid anesthesia, to follow in real time the needle movement. The most significant pitfall of the US-guided technique, even after appropriate training, is the time required for the ultrasound itself, since patient must maintain positioning longer than the traditional blind technique.

Other confounding factors are the wrong identification of the lumbosacral junction, and the missing recognition of junction abnormalities that occur in $12 \%$ of the population. The predominantly sought intervertebral levels are those between L3 and L5. In this way, US has been able to resolve doubts about the correct intervertebral level's identification, by using the sacrum as a starting point to calculate the position of other cranial segments. Once the US scan is finished and sterile gel is removed from the skin, local anesthesia is performed and then subarachnoid anesthesia is performed with a spinal needle introduced at the marked skin target. Regarding the predictive role of the US system, we have drawn conclusions about the correlations between the most influential risk factors for a "difficult spinal puncture" (spinal deformity and amplitude of intervertebral spaces) and US assistance. Regarding column anatomical alterations, the US contributes to adequately identify the midline and intervertebral space, reducing external anatomical confounders and allowing the correct needle orientation.

In cases of reduced intervertebral spaces, another factor that affect the success of a subarachnoid anesthetic, US might be superior to blind technique for two reasons. Firstly, manual palpation alone provides only a rough estimate of the passage through which the spinal needle will passed. Very often, the real perception of the width of the space comes at the very moment in which the spinal needle is aligned or ongoing while the introducer is inserted. The operator can therefore under or overestimate the space amplitude, but recognize a space narrowing only when the procedure started. In summary, there could be a mismatch between the parameter recorded before the procedure and the real width of the intervertebral space. Secondly, the US anatomical study helps the operator to view and calculate intervertebral space width, ratios and inclinations of the spinous processes and the articular processes, to identify the needle insertion point, estimating its real dimensions before the procedure. The most obvious limitation of the US-technique for a neurassial block is represented by the increased overall procedural time, defined as the time between the preparation of the sterile field and the end of the procedure.

Moreover, the US scan performed with a sterile probe cover needs several minutes more than the blind technique, while keeping the patient in an adequate position for the entire procedure. The prolongation of US scan, especially in difficult anatomical cases or for reasons of inexperience, could lead to patient discomfort or compromise his correct positioning at the time of puncture. These factors that must be carefully considered and monitored. However, comparing timing differences between the two techniques, analyzing the needle insertion time and the liquor obtaining, US is faster as compared to the blind technique and compensate in a certain way the longer time required for the US study of the column. Moreover, it should be mentioned that the increase in non-operating time can be overcome by a totally ecoguided approach with introducer-free spinal needles, such as the Quincke, performing US scan and puncture together, thus ensuring US viewing together with a reduced overall procedural time. In our clinical practice we use primarily an atraumatic needle such as the 25G Whitacre, suitable for US-guided method. This choice, reducing the risks of dura mater trauma, ensure a greater patient safety together with pre-procedural US guidance.

\section{Conclusion}

In conclusion, studies with larger sample size are necessary to analyze further implications and aspects on the use of spinal ultrasonography and the efficacy of neurassial blocks. In support of these studies, especially in a future perspective of a more systematic approach, the US of the neurassial district showed to be an accurate and valid aid for subarachnoid anesthesia in terms of success and safety.

\section{References}

1. Kijinn Chin, Manoj Kumar Karmakar, Philip Peng (2011) Ultrasonography of the Adult Thoracic and Lumbar Spine for Central Neuraxial BlockadeAnesthesiology 114(6): 1459-1485.

2. Karmakar MK (2009) Ultrasound for central neuraxial blocks. Techniques in Regional Anesthesia and Pain Management 13(3): 161170.

3. Perlas A (2010) Evidence for the use of ultrasound in Neuraxial blocks. Reg Anesth Pain Med 35(2): S43-46.

4. Halpern SH, Banerjee A, Stocche R, Glanc P (2010) The use of ultrasound for lumbar spinous process identification: A pilot study. Can J Anaesth 57(9): 817-822.

5. Chin KJ, Chan VW, Ramlogan R, Perlas A (2010) Real-time ultrasoundguided spinal anesthesia in patients with a challenging spinal anatomy: Two case reports. Acta Anaesthesiol Scand 54(2): 252-255.

6. Whitty R, Moore M, Macarthur A (2008) Identification of the lumbar interspinous spaces: Palpation versus ultrasound. Anesth Analg 106(2): 538-540.

7. Carvalho JC (2008) Ultrasound-facilitated epidurals and spinals in obstetrics. Anesthesiol Clin 26(1): 145-158.

8. Chin KJ, Perlas A, Singh M, Arzola C, Prasad A, et al. (2009) An ultrasound-assisted approach facilitates spinal anesthesia for total joint arthroplasty. Can J Anaesth 56(9): 643-650. 
9. Amer Majeed, Iftikhar Ahmed, Ghadah Jamaan Alkahtani, Nasser Abdullah Altahtam (2017) Ultrasound-guided continuous spinal anesthesia for cesarean section in a parturient with scoliosis corrected with Harrington's rod surgery-Saudi J Anaesth 11(4): 479-482.

10. Chin KJ, Macfarlane AJ, Chan V, Brull R (2009) The use of ultrasound to facilitate spinal anesthesia in a patient with previous lumbar laminectomy and fusion: A case report. J Clin Ultrasound 37(8): 482-485

11. Lee PJ, Tang R, Sawka A, Krebs C, Vaghadia H (2011) Brief report: Realtime ultrasound-guided spinal anesthesia using Taylor's approach. Anesth Analg 112(5): 1236-1238.

12. Perlas A (2010) Evidence for the use of ultrasound in neuraxial blocks. Reg Anesth Pain Med 35(2 Suppl): S43-S46.

13. Perlas Anahi, Chaparro Luis E, Chin Ki Jinn (2016) Lumbar Neuraxial Ultrasound for Spinal and Epidural Anesthesia: A Systematic Review and Meta-Analysis-Regional Anesthesia and Pain Medicine 41(2): 251-260.

14. Vallejo MC, Phelps AL, Singh S, Orebaugh SL, Sah N (2010) Ultrasound decreases the failed labor epidural rate in resident trainees International journal of obstetric anesthesia 19(4): 373-378.

15. Chin KJ, Ramlogan R, Arzola C, Singh M, Chan V (2013) The utility of ultrasound imaging in predicting ease of performance of spina anesthesia in an orthopedic patient population. Reg Anesth Pain Med 38(1): 34-38

16. Grau T, Leipold RW, Conradi R, Martin E (2001) Ultrasound control for presumed difficult epidural puncture. Acta Anaesthesiol Scand 45(6): 766-771.

17. Geng J, Chen XL, Wang XD, Guo XY, Li M (2016) Ultrasound imaging increases first-attempt success rate of neuraxial block in elderly patients-Nov 22. Zhonghua Yi Xue Za Zhi 96(43): 3459-3463.

18. Shaikh F, Brzezinski J, Alexander S (2013) Ultrasound imaging for lumbar punctures and epidural catheterisations: systematic review and metaanalysis. BMJ pp. 346.

19. Yamauchi M, Honma E, Mimura M, Yamamoto H, Takahashi E, et al. (2006) Identification of the lumbar intervertebral level using ultrasound imaging in a post-laminectomy patient. J Anesth 20(3): 231-233.

20. Chin KJ, Macfarlane AJ, Chan V, Brull R (2009) The use of ultrasound to facilitate spinal anesthesia in a patient with previous lumbar laminectomy and fusion: A case report. J Clin Ultrasound 37(8): 482-485.

21. Ki Jinn Chin, Anahi Perlas, Vincent Chan, Danielle Brown Shreves, Arkadiy Koshkin, et al. (2011) Ultrasound Imaging Facilitates Spinal Anesthesia in Adults with Difficult Surface Anatomic Landmarks. Anesthesiology 115(1): 94-101.

22. Margarido CB, Arzola C, Balki M, Carvalho JC (2010) Anesthesiologists' learning curves for ultrasound assessment of the lumbar spine. Can J Anaesth 57(2): 120-126.

23. Halpern SH, Banerjee A, Stocche R, Glanc P (2010) The use of ultrasound for lumbar spinous process identification: A pilot study. Can J Anaesth 57(9): 817-822.

24. Porter RW, Wicks M, Ottewell D (1978) Measurement of the spinal canal by diagnostic ultrasound. J Bone Joint Surg Br 60-B(4): 481-484.

25. Cork RC, Kryc JJ, Vaughan RW (1980) Ultrasonic localization of the lumbar epidural space. Anesthesiology 52(6): 513-516.
26. Currie JM (1984) Measurement of the depth to the extradural space usingultrasound. Br J Anaesth 56(4): 345-347.

27. Reynolds F (2001) Damage to the conus medullaris following spinal anaesthesia. Anaesthesia 56(3): 238-247.

28. Schlotterbeck H, Schaeffer R, Dow WA, Touret Y, Bailey S, et al. (2008) Ultrasonographic control of the puncture level for lumbar neuraxial block in obstetric anaesthesia. Br J Anaesth 100(2): 230-234.

29. Whitty R, Moore M, Macarthur A (2008) Identification of the lumbar interspinous spaces: palpation versus ultrasound. Anesth Analg 106(2): 538-540.

30. Locks GF, Almeida MC, Pereira AA (2010) Use of the ultrasound to determine the level of lumbar puncture in pregnant women. Rev Bras Anestesiol 60(1): 13-19.

31. Broadbent CR, Maxwell WB, Ferrie R, Wilson DJ, Gawne Cain M, et al. (2000) Ability of anaesthetists to identify a marked lumbar interspace. Anaesthesia 55(11): 1122-1126.

32. Li JW, Karmakar MK, Li X, Kwok WH, Ngan Kee WD (2011) Gelatin-agar lumbosacral spine phantom: a simple model for learning the basic skills required to perform real-time sonographically guided central neuraxial blocks. J Ultrasound Med 30(2): 263-272.

33. Chin KJ, Tse CC, Chan V (2011) Practical considerations in preparing the pig carcass spine phantom. Reg Anesth Pain Med 36(1): 91-92.

34. Kim JT, Bahk JH, Sung J (2003) Influence of age and sex on the position of the conus medullaris and Tuffier's line in adults. Anesthesiology, Dec 99(6):1359-1363

35. Balki M, Lee Y, Halpern S, Carvalho JC (2009) Ultrasound imaging of the lumbar spine in the transverse plane: the correlation between estimated and actual depth to the epidural space in obese parturients. Anesth Analg 108(6): 1876-1881.

36. Grau T (2003) The evaluation of ultrasound imaging for neuraxial anesthesia. Can J Anaesth 50(1s): R1-R8.

37. Karmakar MK, Li X, Kwok WH, Ho AM, Ngan Kee WD (2012) Sonoanatomy relevant for ultrasound-guided central neuraxial blocks via the paramedian approach in the lumbar region. Br J Radiol 85(1015): e262-e269.

38. Furness G, Reilly MP, Kuchi S (2002) An evaluation of ultrasound imaging for identification of lumbar intervertebral level. Anaesthesia 57(3): 277 280.

39. Capdevila X, Dadure C (2004) Perioperative management for one day hospital admission: Regional anesthesia is better than general anesthesia. Acta Anaesthesiol Belg 55(Suppl): 33-36.

40. Horlocker TT, Mc Gregor DG, Matsushige DK, Schroeder DR, Besse JA (1997) A retrospective review of 4767 consecutive spinal anesthetics: central nervous system complications. Perioperative Outcomes Group. Anesth Analg 84(3): 578-584.

41. Chin KJ, Chan V (2010) Ultrasonography as a preoperative assessment tool: Predicting the feasibility of central Neuraxial blockade. Anesth Analg 110(1): 252-253. 
ISSN: 2574-1241

DOI: $10.26717 /$ BJSTR.2019.23.003880

De Cosmo Germano. Biomed J Sci \& Tech Res

(C) (P) This work is licensed under Creative

Submission Link: https://biomedres.us/submit-manuscript.php

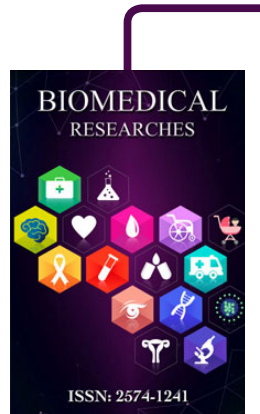

Assets of Publishing with us

- Global archiving of articles

- Immediate, unrestricted online access

- Rigorous Peer Review Process

- Authors Retain Copyrights

- Unique DOI for all articles

https://biomedres.us 